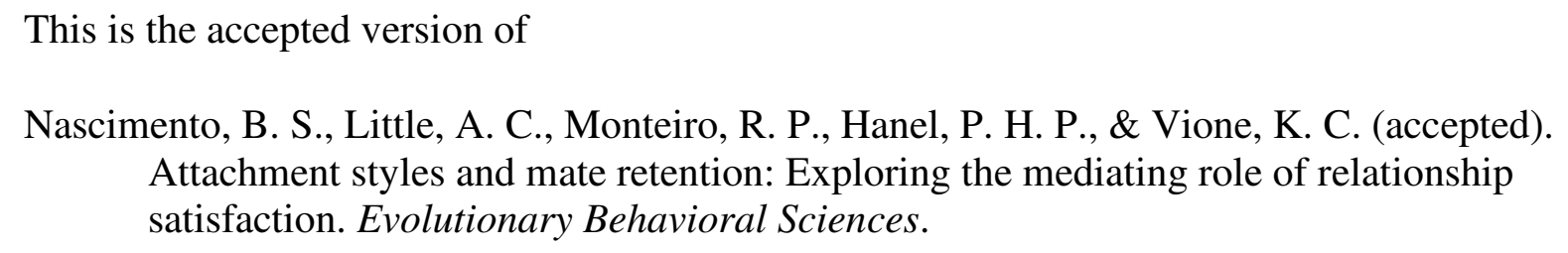

Title: Attachment styles and mate retention: Exploring the mediating role of relationship satisfaction

Running head: Attachment and mate retention

Conflict of interests: None

*Corresponding author. Phone number: 447478216015. Address: Kingston Lane, UB8 3PN

41 Funding: Capes Foundation, Ministry of Education - Brazil (99999.001967/2015-00).

42 Acknowledgements: This research was funded by Capes Foundation, Ministry of Education

43 - Brazil (99999.001967/2015-00). 
1 Ethics approval: All procedures performed in this study were in accordance with the ethical

2 standards of the psychology ethics committee of the University of Bath (ethical approval

3 code: $17-218)$.

4 Availability of data and materials: The data and materials used in the research are 5 available upon request. The data and materials can be obtained by emailing Bruna

$6 \quad$ Nascimento at nascimento.brunads@gmail.com.

7

8

9

10

11

12

13

14

15

16

17

18 


\title{
Attachment styles and mate-retention: Exploring the mediating role of relationship satisfaction
}

\begin{abstract}
This study investigated whether relationship satisfaction mediates the association between attachment styles and mate-retention strategies. Four-hundred and twenty individuals in a heterosexual committed relationship participated in this study $\left(79.7 \%\right.$ women; $M_{\text {age }}=$ 23.22; $S D_{\text {age }}=8.07$ ). Participants completed questionnaires assessing attachment styles, relationship satisfaction, and mate-retention strategies. The results replicated previous findings by showing that insecure attachment is positively associated with benefit-provisioning and cost-inflicting mate-retention strategies and extended previous research by showing that relationship satisfaction mediates these associations. The present findings confirm evolutionary predictions on romantic relationships that relationship satisfaction may serve as a monitor determining how individuals act to preserve their relationships.
\end{abstract}

Keywords: mate retention, attachment styles, relationship satisfaction.

Public Significance Statement. This study found that, although individuals who are insecurely attached tend to feel unhappy with their relationship, they still attempt to retain their partners. However, insecurely attached individuals do this by using negative strategies, while minimising the display of positive ones. These findings suggest that reducing attachment anxiety and avoidance could increase relationship satisfaction and as a result, prevent the use of negative strategies in the relationship.

\section{Introduction}

Long-term romantic relationships are a central part of people's lives with benefits such as emotional and social support. However, relationships are threatened by incompatibility and arguments with the partner, as well as infidelity, which often results in relationship termination (Bravo et al., 2017). Having faced such threats recurrently in the evolutionary past, humans have developed mate-retention strategies to safeguard their relationships (Buss, 1998). Materetention strategies may be grounded in feelings of insecurity and, as such, activated by cues to infidelity or may be due to desiring increased partner's commitment (Campbell \& Ellis, 2005). Given that attachment styles guide an interpersonal orientation in close relationships (Hazan \& Shaver, 1987), attachment styles have been found to play a role in partner retention (Barbaro et al., 2016; Barbaro et al., 2018).

However, there is limited research addressing the link between attachment styles and mate-retention strategies. Recent evidence found that perceived risk of infidelity mediates the 
1 association between attachment anxiety and cost-inflicting mate retention (Barbaro et al.,

2 2019), whereas aggressive and non-assertive communication styles mediate the association

3 between attachment style and jealousy induction (Wegner et al., 2018). These findings suggest

4 that attachment styles influence the frequency of mate retention by influencing multiple

5 relationship domains. Given that anxiously and avoidantly attached individuals tend to be less

6 satisfied with their relationships and that relationship satisfaction, as a mechanism that

7 monitors relationship quality (Conroy-Beam et al., 2015), might influence the investment into

8 a relationship, relationship satisfaction could be a potential mediator of the association between

9 attachment styles and mate retention. However, this potential indirect pathway has not been

10 examined in previous research. Therefore, in this study, we further explore the link between

11 attachment styles and mate-retention strategies and builds on previous research by examining

12 the potential mediating role of relationship satisfaction.

$13 \quad 1.1$ Attachment style and relationship satisfaction

14 Attachment theory postulates that children develop working models in interactions with their

15 parents that serve to guide their perception of the social world and manage their interpersonal

16 relationships throughout life (Hazan \& Shaver, 1987). Ainsworth et al. (2015) identified three

17 attachment styles based on the infants reactions to distress: (1) anxious/ambivalent:

18 demonstrate anger and protest towards their primary caregiver; (2) avoidant: avoid their

19 caregiver and show detachment; and (3) secure: use their caregiver as a source of support. The

20 first two are commonly classified as insecure attachment. An individual's attachment extends

21 from their relationship with their primary caregiver, affecting subsequent meaningful

22 relationships in their life (Ainsworth et al., 2015; Bowlby, 1980). Based on this theory, anxious

23 individuals tend to fear rejection and abandonment, and to be vigilant for signs of disinterest

24 or betrayal (Ainsworth et al., 2015; Bowlby, 1980). As such, highly anxious individuals tend

25 to distrust their partners, anticipate partner infidelity, and be more jealous (Toplu-Demirtas et 
1 al., 2020). In turn, avoidant individuals tend to express their need for comfort by isolating

2 themselves, have low expectations for their partners, are unlikely to act with aggression

3 (Fournier et al., 2011), are less committed to their partners, invest less in their romantic

4 relationships, and are less responsive to their partner's needs (Wardecker et al., 2016).

5 Simpson (1990) conducted a longitudinal study with 144 dating couples to investigate

6 the influence of attachment styles on relationship satisfaction and emotions. In the first part of

7 the study, participants answered to measures of their level of trust, interdependence,

8 commitment, emotions experienced in the relationship, and relationship satisfaction. Six

9 months later, participants were contacted via telephone and asked whether they were still dating

10 and the level of distress of those who had dissolved the relationship. Results from the first

11 phase indicated that participants who scored higher on secure attachment reported higher

12 relationship satisfaction, whilst participants who scored higher on avoidant attachment reported

13 lower satisfaction. Regarding anxious attachment, there was a negative association with

14 relationship satisfaction for men only. In the follow-up, $36.36 \%$ of the couples were no longer

15 dating and male participants who scored higher on avoidant attachment experienced less

16 distress following the end of the relationship.

17 The negative association between insecure attachment styles (anxious and avoidant)

18 with relationship satisfaction has been corroborated in meta-analyses (Candel \& Turliuc, 2019;

19 Hadden et al., 2014; Li \& Chan, 2012). Two of these meta-analyses found that the link between

20 avoidant attachment and relationship satisfaction was stronger than the link between anxious

21 attachment and satisfaction (Candel \& Turliuc, 2019; Li \& Chan, 2012). Moreover, in the most

22 recent meta-analysis, Candel and Turliuc (2019) noted that this link between anxious

23 attachment and relationship satisfaction was stronger for married individuals, and the link

24 between avoidance and satisfaction was weaker in older participants and those in longer

25 relationships. 
2 Mate-retention strategies serve to reduce the risk of partner infidelity and relationship

3 dissolution (Buss, 1988). These are divided into cost-inflicting strategies that function by

4 inflicting costs on a partner or on the relationship, and benefit-provisioning strategies that

5 enhance partner's relationship satisfaction (Buss, et al., 2008). Cost-inflicting strategies may

6 involve tactics from monitoring the partner's steps to violence towards a rival. In turn,

7 benefit-provisioning strategies involve more desirable behaviours such as complimenting the

8 partner or appearance enhancement to please the partner.

Multiple variables are associated with mate-retention strategies. For example,

10 individuals with more attractive partners (Nascimento \& Little, 2019) and lower self-esteem

11 (Holden et al., 2014) tend to engage more often in cost-inflicting mate retention. Mate-

12 retention strategies may also be a reaction to threats of abandonment and separation (Fournier

13 et al., 2011). For example, Barbaro et al. (2016) observed that anxious individuals report

14 more jealousy and adopt mate retention strategies to address the risk of infidelity, whilst

15 avoidant individuals adopt mate retention strategies less frequently, which can be understood

16 by their partner as giving less attention. Further evidence found that anxiously attached

17 individuals tend to engage in both cost-inflicting and benefit-provisioning strategies to retain

18 their partners (Barbaro, et al. 2018). On the other hand, avoidant individuals tend to engage

19 less often in both types of mate-retention strategies. However, a recent study only partially

20 confirmed previous results as the positive association between anxious attachment and

21 benefit-provisioning strategies did not replicate (Altgelt \& Meltzer, 2019). Therefore, further

22 investigation of the association between attachment styles and mate-retention strategies is

23 necessary.

24 1.3. Relationship satisfaction and mate retention strategies 
1 Some relationships will provide more benefits than others and so we can expect mechanisms

2 that allow individuals to weight up the benefits against the costs associated with a

3 relationship (Shackelford \& Buss, 1997). Consistent with this view, relationship satisfaction

4 may be an evolved psychological mechanism that tracks the costs and benefits of a romantic

5 relationship (Conroy-Beam et al., 2015). Indeed, individuals that are happier with their

6 relationships tend to be more committed to (Webster et al., 2014), invest more in their

7 relationships (Conroy-Beam et al., 2015), risk more from losing their partners (Shackelford \&

8 Buss, 2000), and are thus less likely to end their relationship (Balsam et al., 2017). Therefore,

9 higher relationship satisfaction should be associated with higher efforts to retain a partner,

10 whereas relationship dissatisfaction would motivate an individual to change the current

11 relationship or find a more advantageous one.

Although few studies explored the link between relationship satisfaction and mate retention specifically, a recent study (Conroy-Beam et al., 2016) observed that individuals

14 who had lower mate value than their partner and their partner had higher mate value in

15 comparison to alternative mates, were more satisfied with their relationship and more likely

16 to perform mate retention behaviours. Adding to this, previous evidence suggests that

17 individuals who are less satisfied with their relationship tend to invest less in the relationship

18 and are more likely to commit infidelity (Lacker et al., 2020), further supporting the

19 assumption that relationship satisfaction monitors costs and benefits of relationships.

201.4 Relationship satisfaction as a mediator of the association between attachment styles and

\section{1 mate-retention strategies}

22 Insecure (i.e., anxious and avoidant) individuals tend to endorse negative beliefs about their

23 relationships (Stackert \& Bursik, 2003) and be less satisfied with it (Candel \& Turliyc, 2019).

24 Highly anxious individuals tend to distrust their partners, anticipate partner infidelity, and be

25 more jealous (Toplu-Demirtas et al., 2021), whereas highly avoidant individuals are less 
1 committed to their partners, invest less in their romantic relationships, and are less responsive

2 to their partner's needs (Wardecker et al., 2016).

3 Consistent with previous research (e.g., Barbaro, et al. 2018), we anticipate that anxious

4 attachment will be positively associated with cost-inflicting and benefit-provisioning

5 strategies, whereas avoidant attachment will be negatively associated with both types of

6 mate-retention strategies. Because relationship satisfaction, which is associated with insecure

7 attachment (Candel \& Turliyc, 2019) is hypothesised to be a mechanism that monitors the

8 quality of a relationship and as such, predicts investment in the relationship (e.g., efforts to

9 retain a desired mate; Conroy-Beam et al., 2016), we hypothesise that relationship

10 satisfaction mediates the association between attachment insecurity and mate retention. We

11 did not create any hypotheses regarding secure attachment as previous studies have found this

12 attachment dimension to be uncorrelated with mate-retention strategies (see Altgelt \&

13 Meltzer, 2019; Barbaro, et al. 2018).

\section{2. Method}

15 2.1 Procedure. Participants were recruited through the Research Participation Scheme from

16 the Department of Psychology, University of Bath, social media (e.g., Facebook), and

17 research advertising websites. The study took place online on Qualtrics. Participants initially

18 read the information sheet, and after giving their informed consent, they completed self-

19 report questionnaires detailed below. Participants were then redirected to a debriefing page,

20 with a more detailed description of the study.

212.2 Participants. We recruited 420 individuals in committed heterosexual relationships, aged between 16 and 77 years $(M=23.22 ; S D=8.07), 79.7 \%$ female, $44.7 \%$ were in a relationship

23 for less than a year (40.4\% in a relationship for less than five years; and $14.9 \%$ for over five

24 years). Participants were either in a relationship or engaged $(83.1 \%)$ or married or cohabiting

25 (16.9\%). Participants were North American (49.5\%), European (27.9\%), Asian (11.7\%), 
1 Latin American (9.7\%), and African or Middle Eastern (1.2\%). A sensitivity analysis using

2 G*Power 3.1 (Faul et al., 2009) revealed that our sample size of 420 participants would be

3 sufficient to detect a small-to-medium effect size of $r=.14$ with a power of .80 (Cohen, 4 1992).

\section{$5 \quad 2.3$ Materials}

6 Mate Retention Inventory (Short-Form) - MRI-SF (Buss et al., 2008). The MRI-SF measures

7 two broad categories of mate retention: cost-inflicting strategies (22 items; e.g., "snooped

8 through my partners personal belongings") and benefit-provisioning strategies (16 items;

9 e.g. "displayed greater affection for my partner"). Participants indicate how often they

10 performed each behaviour within the past year, using a scale varying from 0 (never) to 3

11 (often performed this act). We calculated composite scores for cost-inflicting $(\alpha=.88)$ and

12 benefit-provisioning $(\alpha=.83)$ strategies.

13 Couples Satisfaction Index (Funk \& Rogge, 2007). Participants indicate to what extent each

14 of the items represent how they feel in their relationship (16 items; e.g., "I still feel a strong

15 connection with my partner"). The statements are answered on a 6-point Likert Scale ( $0=$ not

16 true at all to $5=$ completely true), except question one, which was answered on a 7-point

17 Likert Scale $(0=$ extremely unhappy to $6=$ perfect; Please indicate the degree of happiness,

18 all things considered, of your relationship). We calculated a composite score of relationship

19 satisfaction $(\alpha=.94)$.

20 Adult Attachment Scale (Collins \& Read, 1990). The scale evaluates three different

21 dimensions: (a) secure (5 items; e.g., "I am comfortable depending on others"); (b) avoidant

22 (7 items; e.g., "I find it difficult to trust others completely"); and (c) anxiety (7 items; e.g., "I

23 often worry my partner will not want to stay with me"). Participants answered these items

24 using a 5-point scale varying from $1=$ not at all characteristic of me to $5=$ very characteristic 
1 of me. We calculated composite scores for the dimensions secure $(\alpha=.70)$, avoidant $(\alpha=$

$2 \quad .70)$, and anxious $(\alpha=.84)$.

\section{Results}

\subsection{Data Analysis}

5 Descriptive statistics and Pearson correlations were calculated using SPSS version 26. Next,

6 the PROCESS macro for SPSS (Hayes, 2013; model 4) was applied to examine the mediating

7 effect of relationship satisfaction on the association between attachment styles and mate-

8 retention strategies. Avoidant and anxious attachment styles were entered as independent

9 variables, whereas relationship satisfaction was entered as the mediator, and cost-inflicting

10 and benefit-provisioning strategies were entered as dependent variables in the model. The

11 model controlled for age, sex (dummy coded, $0=$ male, $1=$ female), nationality (dummy

12 coded, $0=$ North American, $1=$ Other), relationship status (dummy coded, $0=$ in a

13 relationship, $1=$ married), and relationship length. All continuous variables were

14 standardised prior to the analysis. Mediation analysis were tested with 5000 bootstrap

15 iterations to compute the $95 \%$ confidence intervals. The criterion for mediation was the

16 presence of a significant indirect effect (Rucker et al., 2011).

17 3.2. Correlation analysis

18 Preliminary correlations were calculated between mate retention strategies, attachment styles,

19 and relationship satisfaction, controlling for age, sex, nationality, relationship status, and

20 relationship length. Benefit-provisioning mate-retention strategies were positively correlated

21 with relationship satisfaction $(r=.24, p<.001)$ and anxious attachment $(r=.15, p<.001)$,

22 and negatively correlated with avoidant attachment $(r=-.11, p=.01)$. In turn, cost-inflicting

23 mate retention strategies were negatively correlated with relationship satisfaction $(r=-.27, p$

$24<.001)$, and positively correlated with anxious $(r=.28, p<.001)$ and avoidant attachment $(r$

$25=.10, p=.03)$. 


\section{Table 1}

Correlations between mate-retention strategies, relationship satisfaction, and attachment styles

$\begin{array}{llllll}1 & 2 & 3 & 4 & 5 & \mathrm{M}(\mathrm{SD})\end{array}$

$\begin{array}{llllll}\text { 1. Benefit- } & .42^{* *} & .24^{* *} & 0 & -.11^{*} & 2.68(.48)\end{array}$

provisioning

2. Cost-

inflicting

$.40^{* *}$

$-.27^{* *}$

$-.03$

.07

$1.60(.83)$

3. Relationship

satisfaction

$$
.24^{* *}-.31^{* *}
$$

.02

$-.11^{*}$

$.15^{* *}$
$-.02$

$.10^{*}$

$.28^{* *}$

$$
.11^{*}
$$$$
-.25^{* *}
$$

6. Anxious

.15

Notes. ${ }^{*} p<.001,{ }^{*} p<.05$. Partial correlations control for age, sex, relationship status, and relationship length. Zero-order correlations are displayed above the diagonal.

\subsection{Mediation Analysis}

Benefit-provisioning strategies. The model explained approximately $18 \%$ of variance

11 in benefit-provisioning strategies $\left[R^{2}=.18, F(8,400)=11.02, p<.001\right]$. A positive direct

effect of anxious attachment and a negative direct effect of avoidant attachment on benefitprovisioning strategies were found (see Figure 1). Analysis also revealed an indirect effect of anxious attachment on benefit-provisioning $(b=-.05$, BootSE $=.02,95 \%$ BootCI [-.091, .018]) through relationship satisfaction, confirming our prediction that relationship satisfaction functions as a mediator. An indirect effect of avoidant attachment on benefit-

17 provisioning $(b=-.04$, BootSE $=.02,95 \%$ BootCI [-.076, -.011]) through relationship satisfaction was also found. The overall effect size of this model was medium $\left(f^{2}=0.21\right.$;

19 Cohen, 1988). Of the covariates, only age ( $b=-.09,95 \%$ CI [-.16, -.02]) and relationship

20 length $(b=.13,95 \%$ CI $[.07, .18])$ were associated with benefit provisioning strategies.

21 However, the model with and without the covariates produced almost identical results. 
2 cost-inflicting strategies $\left[R^{2}=.18, F(8,400)=8.90, p<.001\right]$. Positive direct effects of

3 anxious attachment on cost-inflicting strategies were found (see Figure 1). Analysis also

4 revealed an indirect effect of anxious attachment on cost-inflicting strategies $(b=.06$,

5 BootSE $=.02,95 \%$ BootCI $[.023, .094])$ through relationship satisfaction, confirming our

6 predictions. An indirect effect of avoidant attachment on cost-inflicting strategies $(b=.04$,

7 BootSE $=.02,95 \%$ BootCI $[.014, .079])$ through relationship satisfaction was also found.

8 The overall effect size of this model was medium $\left(f^{2}=0.21\right.$; Cohen, 1988). Of the covariates,

9 only age $(b=-.11,95 \%$ CI [-.20, -.03]), sex $(b=-.16,95 \%$ CI [-.28, -.03]) and relationship

10 length $(b=.09,95 \%$ CI $[.03, .15])$ were associated with benefit provisioning strategies.

11 However, the model with and without the covariates produced almost identical results.

12

13 Figure 1

14 Mediation Model

15

16

17

18

19

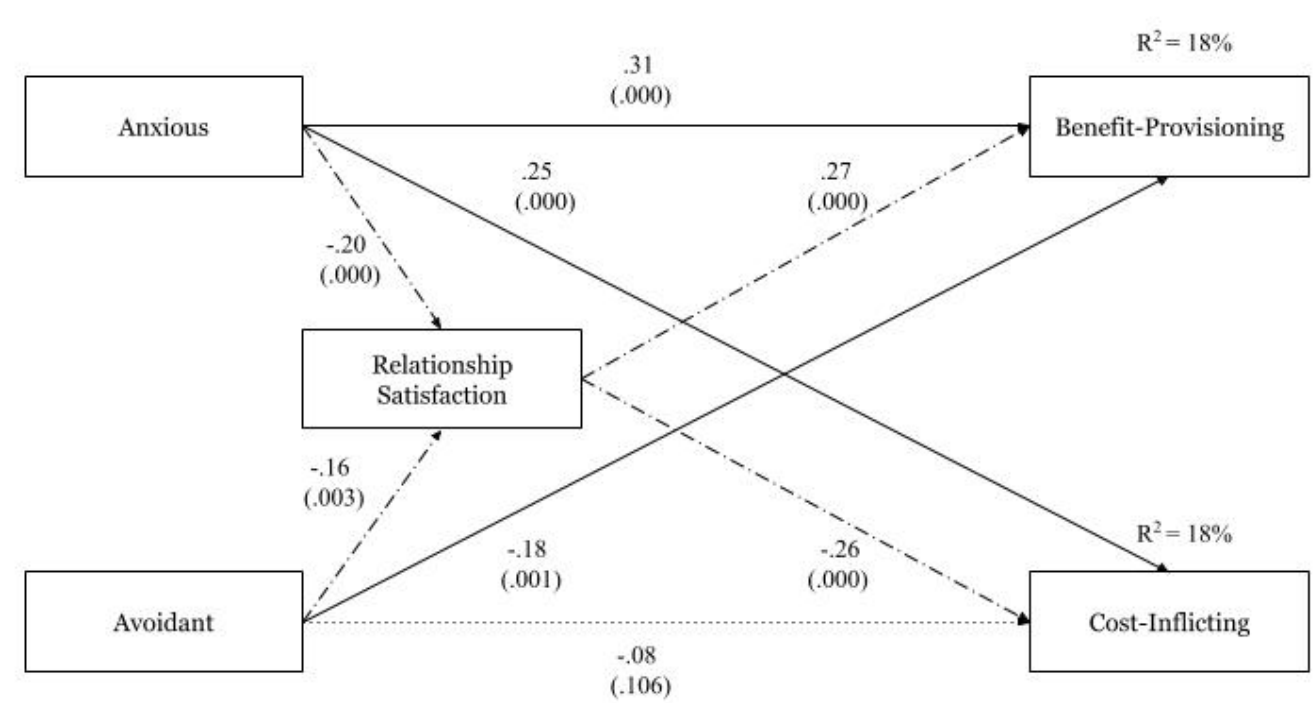

Note. Dashed lines represent significant indirect effects of anxious attachment on benefit-provisioning and cost-inflicting mate retention, and of avoidant attachment on benefit-provisioning and cost-inflicting mate retention through relationship satisfaction. The dotted line represents non-significant direct effects. Model controls for age, sex, nationality, relationship length, and relationship status. 
1 This study explored a potential indirect effect of attachment styles on mate-retention

2 strategies through relationship satisfaction. In line with previous literature (Barbaro et al.,

3 2016; Barbaro et al., 2017), this study demonstrated that anxiously attached individuals tend

4 to engage more often in both cost-inflicting and benefit-provisioning strategies. As anxious

5 individuals place greater value on close relationships, but are also afraid of rejection

6 (Ainsworth et al., 1978), they tend, as a consequence, to engage in a series of behaviours that

7 may also be dysfunctional for their relationship (Feeney, 2008). This also reflects on their

8 mate retention behaviours as, probably motivated by their fear of rejection, they end up

9 investing in different types of mate retention strategies to retain their partners.

10 Avoidant individuals reported lower frequency of benefit-provisioning strategies

11 (Barbaro et al., 2016; Barbaro et al., 2018) consistent with a tendency to value independence

12 and avoid intimacy as they engage less in strategies that show their feelings to their partners.

13 We did not confirm previous findings that avoidant individuals are less likely to engage in

14 cost-inflicting strategies. Although there was a simple negative correlation between avoidant

15 attachment and cost-inflicting strategies, in the mediation model, this association was non-

16 significant when controlling for anxious attachment. The association between avoidant

17 attachment and cost-inflicting strategies may be due to its shared variance with anxious

18 attachment. Regarding secure attachment, there was a small positive correlation between this

19 style of attachment and relationship satisfaction, supporting previous research (Hadden et al.,

20 2014). However, we did not find any associations between secure attachment and mate

21 retention strategies, which also corroborates previous studies (Barbaro et al., 2016; Barbaro et

22 al., 2018).

23 Confirming our predictions, our study also found that relationship satisfaction

24 mediates the association between attachment styles and mate retention strategies and so the

25 influence of attachment styles on mate-retention is partially indirect through reduced 
1 relationship satisfaction. Specifically, both anxious and avoidant attachment styles are

2 associated with lower relationship satisfaction that in turn is associated with lower frequency

3 of benefit-provisioning strategies and higher frequency of cost-inflicting strategies. In other

4 words, relationship satisfaction serves as an underlying mechanism of the attachment style-

5 mate-retention strategy link. Indeed, this supports previous studies showing that insecurely

6 attached individuals tend to perceive more conflict in the relationship, have lower trust in

7 their partners, and perceive lower support from their partners, which, which is associated with

8 lower relationship satisfaction (Candel \& Turliuc, 2019). As a monitor of relationship quality,

9 high relationship satisfaction is expected to motivate individuals to preserve and nurture their

10 relationships, whereas low relationship satisfaction is expected to motivate individuals to

11 terminate the relationship (Conroy-Beam et al., 2016). However, partly contradicting our

12 predictions, we found that low relationship satisfaction is associated with higher frequency of

13 cost-inflicting strategies, and lower frequency of benefit-provisioning strategies. Thus, it

14 seems that although individuals who are insecurely attached tend to perceive their

15 relationship as low in quality, they still attempt to retain their partners. However, they attempt

16 to retain their partners by using strategies that involve emotional manipulation and jealousy

17 induction (i.e., cost-inflicting strategies), while reducing the frequency of strategies such as

18 display of love and care (i.e., benefit-provisioning strategies). This also implies that

19 individuals who are happier with their relationship will invest in it by engaging in benefit-

20 provisioning strategies and relying less on cost-inflicting strategies (Conroy-Beam et al.,

21 2015). This may be because cost-inflicting strategies may decrease the quality of a

22 relationship (Altgelt \& Meltzer, 2019) and may also lead the partner to reciprocate with

23 equally negative strategies. These findings suggest that reducing attachment anxiety and

24 avoidance could increase high relationship satisfaction and therefore, prevent the display of

25 cost-inflicting strategies. 
One limitation of this study is that the gender-imbalanced sample did not allow for

2 comparisons across sexes of the mediational patterns found in this study. Future research

3 could investigate how the mediational patterns found vary across sexes because men and

4 women use mate-retention strategies differently, such that men tend to engage more often in

5 strategies such as resource display than women do, whereas women tend to engage more

6 often in strategies such as appearance enhancement in comparison to men (Albert \&

7 Arnocky, 2016). A second limitation of this study is that we relied on the report of one of the

8 partners only. Because the attachment styles of the partner may also play role on an

9 individual's relationship satisfaction and mate retention strategies, this is another area for

10 future studies that could consider reports from both partners. A third limitation is the non-

11 probability and convenience nature (i.e., non-random internet recruitment so participants are

12 self-selected) of the sample, which can limit the generalisability of our findings. Another

13 limitation is that this study relied on cross-sectional data only. Thus, we cannot imply

14 causation. It may be the case, for example, that the association between relationship

15 satisfaction and mate-retention strategies is bidirectional. Longitudinal studies are necessary

16 to clarify the links between the variables explored in this study. The current study only

17 explored mate-retention strategies among heterosexual individuals. Given that sexual

18 orientation influences the performance of mate-retention strategies (Brewer \& Hamilton,

19 2014), future studies should address homosexual relationship dynamics.

20 In conclusion, the current study extends previous findings on the association between

21 attachment styles, relationship satisfaction, and mate-retention strategies. Our findings

22 suggest that the association between attachment styles and mate-retention strategies is not

23 direct but mediated by relationship satisfaction. 
1 Ainsworth, M. D., Blehar, M. C., Waters, E., \& Wall, S. (1978). Patterns of attachment. Hillsdale, NJ: Erlbaum.

3 Ainsworth, M. D., Blehar, M., Waters, E., \& Wall, S. (2015). Patterns of attachment: A psychological study of the stranger situation. New York, NY: Routledge

Altgelt, E. E., \& Meltzer, A. L. (2019). Associations Between Premarital Factors and FirstMarried, Heterosexual Newlywed Couples' Frequency of Sex and Sexual Satisfaction Trajectories. The Journal of Sex Research, 58(2), 1-14. https://doi.org/10.1080/00224499.2019.1695722

Albert, G., \& Arnocky, S. (2016). Use of mate retention strategies. Encyclopedia of Evolutionary Psychological Science, Springer, New York, 01-11. Retrieved from https://evolutionlab.nipissingu.ca/wp-content/uploads/sites/68/2017/09/use-of-materetention-encyclopedia-entry.pdf

Balsam, K. F., Rothblum, E. D., \& Wickham, R. E. (2017). Longitudinal predictors of relationship dissolution among same-sex and heterosexual couples. Couple and Family Psychology: Research and Practice, 6(4), 247-257. https://doi.org/10.1037/cfp0000091

Barbaro, N., Sela, Y., Atari, M., Shackelford, T. K., \& Zeigler-Hill, V. (2019). Romantic attachment and mate retention behavior: The mediating role of perceived risk of partner infidelity. Journal of Social and Personal Relationships, 36(3), 940-956. https://doi.org/10.1177/0265407517749330

Barbaro, N., Pham, M. N., Shackelford, T. K., \& Zeigler-Hill, V. (2016). Insecure romantic attachment dimensions and frequency of mate retention behaviors. Personal Relationships, 23(3), 605-618. https://doi.org/10.1111/pere.12146

Barbaro, N., Sela, Y., Atari, M., Shackelford, T. K., \& Zeigler-Hill, V. (2018). Romantic attachment and mate retention behavior: The mediating role of perceived risk of partner 
infidelity. Journal of Social and Personal Relationships, 36(3), 940-956. https://doi.org/10.1177/0265407517749330

Bravo, V., Connolly, J., \& McIsaac, C. (2017). Why did it end? Breakup reasons of youth of different gender, dating stages, and ages. Emerging adulthood, 5(4), 230-240. https://doi.org/10.1177/2167696817700261

Brewer, G., \& Hamilton, V. (2014). Female mate retention, sexual orientation, and gender identity. Evolutionary Behavioral Sciences, 8(1), 12-19. https://doi.org/10.1037/h0097245

Bowlby, J. (1980). Attachment and loss: Vol. 3. Loss: Sadness and depression. New York: Basic Books.

Buss, D. M. (1988). From vigilance to violence: Tactics of mate retention in American undergraduates. Ethology and Sociobiology, 9, 291-317. https://doi.org/10.1016/01623095(88)90010-6

Buss, D. M., Shackelford, T. K., \& McKibbin, W. F. (2008). The mate retention inventoryshort form (MRI-SF). Personality and Individual Differences, 44(1), 322-334. https://doi.org/10.1016/j.paid.2007.08.013

Campbell, L., \& Ellis, B. J. (2005). Commitment, love, and mate retention. In D. Buss (ed), The Handbook of Evolutionary Psychology (pp. 419-442). John Wiley and Sons Ltd. https://doi.org/10.1002/9780470939376.ch14

Candel, O. S., \& Turliuc, M. N. (2019). Insecure attachment and relationship satisfaction: A meta-analysis of actor and partner associations. Personality and Individual Differences, 147, 190-199. https://doi.org/10.1016/j.paid.2019.04.037

Cohen, J. (1992). A power primer. Psychological Bulletin, 112(1), 155159. https://doi.org/10.1037/0033-2909.112.1.155 
1 Collins, N. L., \& Read, S. J. (1990). Adult attachment, working models, and relationship quality in dating couples. Journal of Personality and Social Psychology, 58(4), 644663. https://doi.org/10.1037/0022-3514.58.4.644

4 Conroy-Beam, D., Goetz, C. D., \& Buss, D. M. (2015). Why do humans form long-term mateships? An evolutionary game-theoretic model. Advances in Experimental Social Psychology, 51, 1-39. https://doi.org/10.1016/bs.aesp.2014.11.001

Conroy-Beam, D., Goetz, C., \& Buss, D. M. (2016). What predicts romantic relationship satisfaction and mate retention intensity? Mate preference fulfillment or mate value discrepancies? Evolution and Human Behavior, 37(6), 440-448. https://doi.org/10.1016/j.evolhumbehav.2016.04.003

Faul, F., Erdfelder, E., Buchner, A., \& Lang, A. G. (2009). Statistical power analyses using G* Power 3.1: Tests for correlation and regression analyses. Behavior Research Methods, 41(4), 1149-1160. https://doi.org/10.3758/BRM.41.4.1149

Feeney, J. A. (2008). Adult romantic attachment: Developments in the study of couple relationships. In J. Cassidy \& P. R. Shaver (Eds.), Handbook of attachment: Theory, research, and clinical applications (p. 456-481). The Guilford Press.

Fournier, B., Brassard, A., \& Shaver, P. R. (2011). Adult attachment and male aggression in couple relationships: The demand-withdraw communication pattern and relationship satisfaction as mediators. Journal of interpersonal violence, 26(10), 1982-2003. https://doi.org/10.1177/0886260510372930

Funk, J. L., \& Rogge, R. D. (2007). Testing the ruler with item response theory: Increasing precision of measurement for relationship satisfaction with the Couples Satisfaction Index. Journal of Family Psychology, 21(4), 572-583. https://doi.org/10.1037/0893$\underline{3200.21 .4 .572}$ 
1 Gleeson, G., \& Fitzgerald, A. (2014). Exploring the Association between Adult Attachment Styles in Romantic Relationships, Perceptions of Parents from Childhood and Relationship Satisfaction. Health, 6(13), 1643-1661. https://doi.org/10.4236/health.2014.613196

Hadden, B. W., Smith, C. V., \& Webster, G. D. (2014). Relationship duration moderates associations between attachment and relationship quality: Meta-analytic support for the temporal adult romantic attachment model. Personality and Social Psychology Review, 18(1), 42-58. https://doi.org/10.1177/1088868313501885

Hazan, C., \& Shaver, P. (1987). Romantic love conceptualized as an attachment process. Journal of Personality and Social Psychology, 52(3), 511524. https://doi.org/10.1037/0022-3514.52.3.511

Holden, C. J., Shackelford, T. K., Zeigler-Hill, V., Miner, E. J., Kaighobadi, F., Starratt, V. G., ... \& Buss, D. M. (2014). Husband's esteem predicts his mate retention tactics. Evolutionary Psychology, 12(3), 147470491401200311.

Lacker, T. J., Walther, A., Waldvogel, P., \& Ehlert, U. (2020). Fatherhood Is Associated with Increased Infidelity and Moderates the Link between Relationship Satisfaction and Infidelity. Psych, 2(4), 370-384. https://doi.org/10.3390/psych2040027

Li, T., \& Chan, D. K. S. (2012). How anxious and avoidant attachment affect romantic relationship quality differently: A meta-analytic review. European Journal of Social Psychology, 42(4), 406-419. https://doi.org/10.1002/ejsp.1842

Nascimento, B.S., \& Little, A.C. (2019). Mate retention Strategies, Self-Esteem, Mate Value and Facial Attractiveness Disparity in Brazil and in the UK. Journal of Sex \& Marital Therapy, 45(6), 461-472. https://doi.org/10.1080/0092623X.2018.1557307 
1 Rucker, D. D., Preacher, K. J., Tormala, Z. L., \& Petty, R. E. (2011). Mediation analysis in social psychology: Current practices and new recommendations. Social and Personality Psychology Compass, 5(6), 359-371. https://doi.org/10.1111/j.1751-9004.2011.00355.x

Shackelford, T. K., \& Buss, D. M. (1997). Marital satisfaction in evolutionary psychological perspective. In R. J. Steinberg \& M. Hojjat (Eds.), Satisfaction in close relationships (pp. 7-25). New York: Guilford Press.

Simpson, J. A. (1990). Influence of attachment styles on romantic relationships. Journal of personality and social psychology, 59(5), 971-980. https://doi.org/10.1037/0022$\underline{3514.59 .5 .971}$

Stackert, R. A., \& Bursik, K. (2003). Why am I unsatisfied? Adult attachment style, gendered irrational relationship beliefs, and young adult romantic relationship satisfaction. Personality and individual differences, 34(8), 1419-1429. https://doi.org/10.1016/S0191-8869(02)00124-1

Toplu-Demirtaş, E., Akcabozan-Kayabol, N. B., Araci-Iyiaydin, A., \& Fincham, F. D. (2020). Unraveling the Roles of Distrust, Suspicion of Infidelity, and Jealousy in Cyber Dating Abuse Perpetration: An Attachment Theory Perspective. Journal of Interpersonal Violence, O(0), 1-31. https://doi.org/10.1177/0886260520927505

Wardecker, B. M., Chopik, W. J., Boyer, M. P., \& Edelstein, R. S. (2016). Individual differences in attachment are associated with usage and perceived intimacy of different communication media. Computers in Human Behavior, 59, 18-27. https://doi.org/10.1016/j.chb.2016.01.029

Wegner, R., Roy, A. R., Gorman, K. R., \& Ferguson, K. (2018). Attachment, relationship communication style and the use of jealousy induction techniques in romantic relationships. Personality and Individual Differences, 129, 6-11. https://doi.org/10.1016/j.paid.2018.02.033 
2

3

4 